\begin{tabular}{|c|l|}
\hline Title & Conductance of a single molecule anchored by an isocy anide substituent to gold electrodes \\
\hline Author(s) & Kiguchi, Manabu; Miura, Shinichi; Hara, Kenji; Sawamura, Masay a; Murakoshi, Kei \\
\hline Citation & $\begin{array}{l}\text { A pplied Physics Letters, 89(213104) } \\
\text { https://doi.org/10.1063/2392816 }\end{array}$ \\
\hline Issue Date & 2006-11-21 \\
\hline Doc URL & http://hdl.handle.net/2115/16949 \\
\hline Rights & Copyright $\odot 2006$ A merican Institute of Physics \\
\hline Type & article \\
\hline File Information & APL89-21.pdf \\
\hline
\end{tabular}

Instructions for use 


\title{
Conductance of a single molecule anchored by an isocyanide substituent to gold electrodes
}

\author{
Manabu Kiguchi, ${ }^{\text {a) }}$ Shinichi Miura, Kenji Hara, Masaya Sawamura, and Kei Murakoshi \\ Division of Chemistry, Graduate School of Science, Hokkaido University, Sapporo 060-0810, \\ Japan
}

(Received 18 August 2006; accepted 6 October 2006; published online 21 November 2006)

\begin{abstract}
The effect of anchoring group on the electrical conductance of a single molecule bridging two Au electrodes was studied using disubstituted [isocyanide $(\mathrm{CN}-)$, thiol $\left(\mathrm{S}_{-}\right)$, or cyanide $(\mathrm{NC}-)$ ] benzene. The conductance of a single $\mathrm{Au} / 1,4$-diisocyanobenzene/Au junction anchored by isocyanide via a $\mathrm{C}$ atom (junction with the $\mathrm{Au}-\mathrm{CN}$ bond) was $3 \times 10^{-3} G_{0}\left(=2 e^{2} / h\right)$. The value was comparable to $4 \times 10^{-3} G_{0}$ of a single Au/1,4-benzenedithiol/Au junction with the Au-S bond. The $\mathrm{Au} / 1,4$-dicyanobenzene/Au molecular junction with the $\mathrm{Au}-\mathrm{NC}$ bond did not show well-defined conductance values. The metal-molecule bond strength was estimated by the distance over which the molecular junction was stretched before breakdown. The stretched length of the molecular junction with the $\mathrm{Au}-\mathrm{CN}$ bond was comparable to that of the Au junction, indicating that the $\mathrm{Au}-\mathrm{CN}$ bond was stronger than the $\mathrm{Au}-\mathrm{Au}$ bond. (C) 2006 American Institute of Physics.
\end{abstract}

[DOI: $10.1063 / 1.2392816$ ]

Recently, molecular electronic devices have attracted attention, since these devices offer the possibility for extreme scale down and ultrasmall energy consumption of logic units. Building the device with a single molecule demands an understanding of the electron transport properties through single molecule junctions connecting two metal electrodes. The electron transport characteristics through a single molecule have been studied using mechanically controllable break junctions ${ }^{1}$ and scanning tunneling microscopy (STM). ${ }^{2-4}$ With regard to anchoring groups of the molecular junction, the $\mathrm{Au}-\mathrm{S}$ bond is used to connect molecules to metal electrodes. It is because the $\mathrm{Au}-\mathrm{S}$ bond is a strong bond, which leads to the effective hybridization of the molecular and metal orbitals. The effective hybridization of the orbitals is essential for the high conductance of the molecular junction. On the other hand, the high conductance of the molecular junction also requires the condition of energy matching between the Fermi level of metal electrodes and conduction orbital of the molecule. ${ }^{5}$ A strong metal-molecule bond and a smaller energy difference are essential for the molecular junction to show high conductance. The energy of the molecular orbital varies with the end group of the molecule. It is important to develop new end groups other than a thiol to enable more stable and higher conductance single molecular junctions.

Among various end groups, isocyanide end groups are attractive. Isocyanides bind to transition metals via a strong coordination bond by effective donation to metal and back donation from metals. The strength of the metal-CN bond is comparable to that of the metal-S bond. Because of this effective interaction between the isocyanide group and metals, air stable isocyanide self-assembled monolayer (SAM) films are formed on $\mathrm{Au}^{6}{ }^{6}$ as with the case of thiol SAMs. ${ }^{7}$ This strong metal-CN bond may contribute to increase the conductance of the junction. Recent theoretical calculations show promise for a molecular junction based on an isocyanide group. ${ }^{8}$ The conductance of the molecular junction

\footnotetext{
${ }^{a)}$ Electronic mail: kiguti@sci.hokudai.ac.jp
}

binding a benzene molecule to $\mathrm{Au}$ atoms by isocyanide coordination (the molecular junction with the $\mathrm{Au}-\mathrm{CN}$ bond) is close to that of the molecular junction with the $\mathrm{Au}-\mathrm{S}$ bond. In the case of $\mathrm{Ni}$ electrodes, the conductance of molecular junctions with isocyanide end groups is higher than those of thiols. ${ }^{8}$ Despite these interests, the conductance of a single molecule with isocyanide end groups has not been studied adequately. $^{9}$

In the present study, we have measured the electrical conductance of several disubstituted $(-\mathrm{S},-\mathrm{NC},-\mathrm{CN})$ benzenes bridging two $\mathrm{Au}$ electrodes. As for the disubstituted benzene, there are only a few reports on the conductance of 1,4-benzenedithiol bridging two Au electrodes. ${ }^{1,3,4}$ Based on the bond strength of the anchoring group, ${ }^{10}$ the conductance of a single molecule was discussed using a simple tunneling model. $^{5}$

A sharp tip was used, comprising a Au wire (diameter $\sim 0.25 \mathrm{~mm},>99.9 \%)$. The substrate was $\mathrm{Au}(111)$, prepared by a flame annealing and quenching method. ${ }^{11}$ The measurements were carried out in tetraethyleneglycol dimethyl ether (tetraglyme) or toluene solutions since isocyanides could be hydrolyzed under aqueous acidic or basic conditions. ${ }^{12}$ The solution contained $1 \mathrm{mM}$ concentrations of 1,4-diisocyanobenzene, 1,4-benzenedithiol, or 1,4-dicyanobenzene. A Au STM tip was repeatedly moved into and out of contact with a $\mathrm{Au}$ (111) substrate at a rate of $50 \mathrm{~nm} / \mathrm{s}$ in the solution. Each molecule was terminated with two isocyanide, thiol, or cyanide end groups bridging the tip and substrate electrodes via the respective anchoring groups, as shown in Fig. 1. Conductance was measured during the breaking process under an applied bias of $20 \mathrm{mV}$ between the tip and substrate. All statistical data were obtained from a large number (over 1000) of individual conductance traces.

Figures 2(a) and 2(b) show the typical conductance traces of $\mathrm{Au}$ point contacts broken in the tetraglyme solution containing $1 \mathrm{mM}$ of 1,4-diisocyanobenzene. The conductance decreased in a stepwise fashion with each step occurring at an integer multiple of $\left(2 \times 10^{-3}-3 \times 10^{-3}\right) G_{0}$. The last plateau before the contact break showed a value of 2 


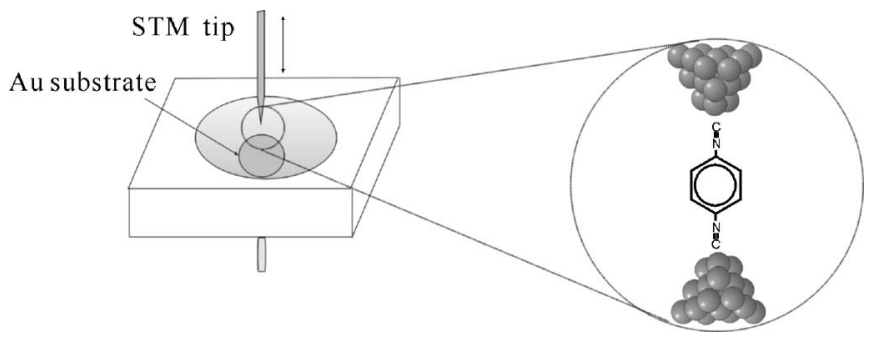

FIG. 1. Schematic illustration of a molecule junction, in which a single molecule is bonded to two Au electrodes.

$\times 10^{-3} G_{0}$. The corresponding histogram shows a feature at $3 \times 10^{-3}\left( \pm 1 \times 10^{-3}\right) G_{0}$. These steps in the conductance trace likely originate from the formation of a stable, single $\mathrm{Au} / 1,4-$ diisocyanobenzene/Au junction, possibly binding a benzene molecule to $\mathrm{Au}$ atoms by isocyanide coordination via $\mathrm{C}$ atoms (molecular junction with the $\mathrm{Au}-\mathrm{CN}$ bond). The conductance behavior in the absence of 1,4-diisocyanobenzene molecules was studied. Neither steps nor peaks were observed in the same conductance regime [see Figs. 2(a) and 2(b)]. The effect of the solvent was also checked using toluene. The inset of Fig. 2(b) shows the conductance histogram observed in toluene solution containing $1 \mathrm{mM}$ of $1,4-$ diisocyanobenzene. The conductance histogram shows a feature near $3 \times 10^{-3} G_{0}$, as is the case with tetraglyme. Based on these experimental results, the conductance of a single molecular junction with the $\mathrm{Au}-\mathrm{CN}$ bond was determined to be $3 \times 10^{-3}\left( \pm 1 \times 10^{-3}\right) G_{0}$.

The conductance of 1,4-dicyanobenzene and 1,4benzenedithiol is discussed. In the $\mathrm{Au} / 1,4-$ dicyanobenzene/Au junction (molecular junction with the $\mathrm{Au}-\mathrm{NC}$ bond), a molecule binds to $\mathrm{Au}$ atoms via the $\mathrm{N}$ atom of the cyanide group, while a molecule binds to Au atoms via the $\mathrm{S}$ atom in the $\mathrm{Au} / 1,4-$ benzenedithiol/Au molecular junction (molecular junction with the $\mathrm{Au}-\mathrm{S}$ bond). Figures 2(c)-2(f) show the conductance traces and histograms of $\mathrm{Au}$ point contacts broken in the tetraglyme solution containing $1 \mathrm{mM}$ of 1,4-dicyanobenzene or 1,4-benzenedithiol. As for 1,4-dicyanobenzene, small conductance steps were observed in the conductance trace. The difference in conductance between the steps was smaller than $5 \times 10^{-4} G_{0}$. The conductance histogram does not show a clear feature above 1 $\times 10^{-3} G_{0}$. Thus, the conductance of the molecular junction with the $\mathrm{Au}-\mathrm{NC}$ bond would be smaller than $1 \times 10^{-3} G_{0}$.
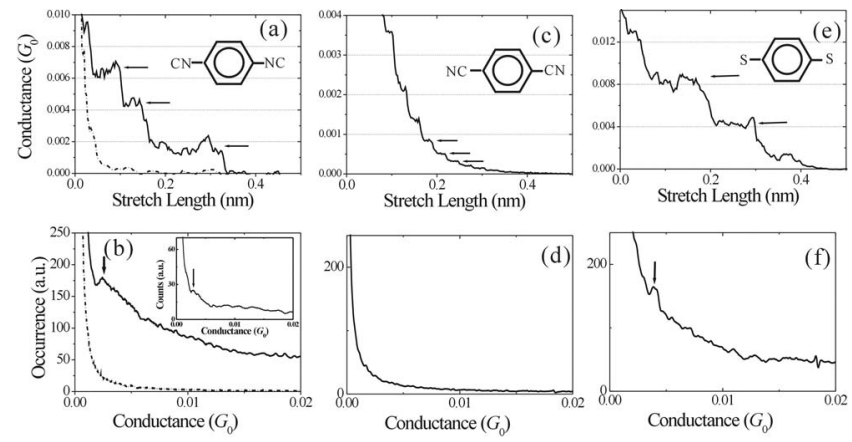

FIG. 2. Typical conductance trace and histogram of Au point contacts broken in the tetraglyme solution consisting of $[(a)$ and (b)] 1,4diisocyanobenzene, [(c) and (d)] 1,4-dicyanobenzene, and [(e) and (f)] 1,4benzenedithiol. The dotted line shows the result in the absence of molecules. The inset of (b) shows the conductance histogram in the toluene solution

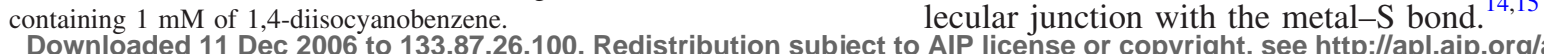

Although another possibility that the molecule could not make stable and reproducible junctions should be also considered for the interpretation on this conductance behavior, estimated value may reflect relatively small conductivity of the junction. For 1,4-benzenedithiol, steps occurring at an integer multiple of $4 \times 10^{-3} G_{0}$ were observed in the conductance traces, and the conductance histogram shows a feature near $4 \times 10^{-3} G_{0}$. Thus, the conductance of a single molecular junction with the $\mathrm{Au}-\mathrm{S}$ bond was determined to be $4 \times 10^{-3}$ $\left( \pm 1 \times 10^{-3}\right) G_{0}$. The conductance of a single molecular junction with the $\mathrm{Au}-\mathrm{S}$ bond was also studied by other groups, and it ranges from $1.1 \times 10^{-2} G_{0}$ (Ref. 13 ) to $4.1 \times 10^{-4} G_{0}{ }^{1}$ Our result of $4 \times 10^{-3} G_{0}$ falls within this range of values. Since the different conductance values for the same molecular junction by orders of magnitude may be due to the different experimental conditions, it is difficult to compare the conductance of single molecular junctions of different molecules measured under different conditions. In the present case, the conductance of a single molecular junction is discussed based on our results measured under the same condition. Statistical analysis of the present data showed that the conductance values of a single molecular junction measured under the same condition could be determined to be in a precision of $30 \%$. Our results showed that the conductance of the molecular junction with the $\mathrm{Au}-\mathrm{CN}$ bond was comparable to the junction with the $\mathrm{Au}-\mathrm{S}$ bond. The conductance of the molecular junction with the $\mathrm{Au}-\mathrm{NC}$ bond was much smaller than that of the junction with the $\mathrm{Au}-\mathrm{S}$ and $\mathrm{Au}-\mathrm{CN}$ bonds.

Our experimental results on the conductance of a single molecular junction are discussed with a simple tunneling model and theoretical calculation results. In the simple tunneling model, ${ }^{5}$ the conductance of a single molecular junction is given by $A(\beta G \rho)^{2}$, where $A, \beta, G, \rho$ are the constant, hopping integral between the molecule and metal, Green's function, and local density of states at the Fermi energy of the contact metal atom, respectively. $\beta$ increases with the bond strength. $G$ decreases with the energy difference between the Fermi level and conduction orbital. Therefore, a strong metal-molecule bond (large $\beta$ ) and small energy difference (large $G$ ) are essential for a molecular junction with high conductance. In the case of the molecular junction with the $\mathrm{Au}-\mathrm{CN}$ bond, the bond strength and the energy difference between the Fermi level and conduction orbital are similar to those with the $\mathrm{Au}-\mathrm{S}$ bond $(\operatorname{similar} \beta$ and $G){ }^{14,15}$ Thus, the conductance of the molecular junction with the $\mathrm{Au}-\mathrm{CN}$ bond would be close to that of the molecular junction with the $\mathrm{Au}-\mathrm{S}$ bond. Theoretical calculations show that the molecular junctions with the $\mathrm{Au}-\mathrm{S}$ bond and $\mathrm{Au}-\mathrm{CN}$ bond have similar conductance values. ${ }^{8}$ Our experimental results agree well with those theoretical estimates. On the other hand, the strength of the Au-NC bond is much smaller than that of the $\mathrm{Au}-\mathrm{CN}$ or $\mathrm{Au}-\mathrm{S}$ bonds, which yields a small $\beta$ for the $\mathrm{Au}-\mathrm{NC}$ bond. Since the energy difference between the Fermi level and conduction orbital of 1,4-dicyanobenzene is close to that of 1,4-diisocyanobenzene, ${ }^{13,14}$ the conductance of the molecular junction with the $\mathrm{Au}-\mathrm{NC}$ bond would be much smaller than that of the molecular junction with the $\mathrm{Au}-\mathrm{CN}$ bond due to a small $\beta$. Theoretical calculations show that the conductance of the molecular junction with the metal-NC bond is three times smaller than that of the molecular junction with the metal-S bond. ${ }^{14,15}$

to AIP license or copyright, see http://apl.aip.org/apl/copyright.jsp 

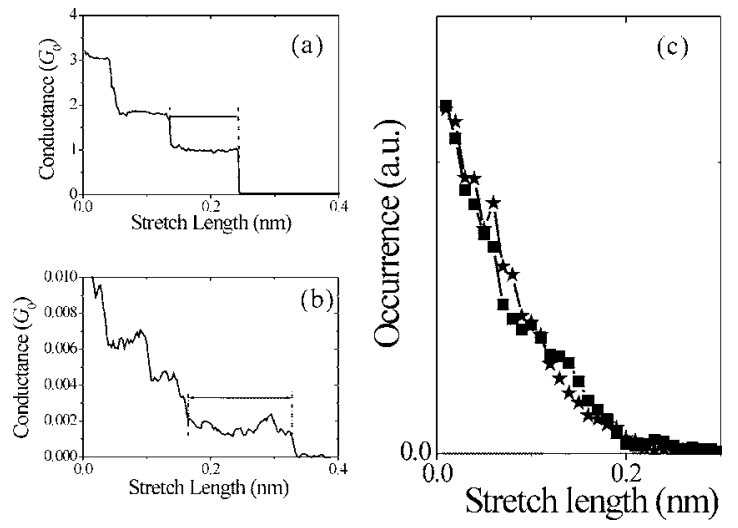

FIG. 3. Typical conductance trace of (a) Au junctions and (b) 1,4diisocyanobenzene junctions in tetraglyme solution containing $1 \mathrm{mM}$ of 1,4diisocyanobenzene. (c) The distribution of lengths for the last conductance plateau for the Au junctions (box) and molecular junctions (star).

Relatively strong binding between $\mathrm{Au}$ and $\mathrm{C}$ atoms in $\mathrm{Au}-\mathrm{CN}$ binding was also demonstrated by conductance traces. Figures 3(a) and 3(b) show the typical conductance traces of $\mathrm{Au}$ point contacts broken in the presence of 1,4diisocyanobenzene. The length of the last conductance plateau of the molecular junction $[0.16 \mathrm{~nm}$ in Fig. 3(b)] was comparable to that of the Au junction [0.12 nm in Fig. 3(a)]. Since the last plateau corresponds to the single molecular or metal atom junction, ${ }^{11}$ the length of the last plateau is the distance over which a molecular or metal junction can be stretched before breakdown (breakdown distance). To evaluate characteristics of the breakdown distance quantitatively, the distributions of the breakdown distance of the Au junctions and molecular junction are shown in Fig. 3(c). The breakdown distance was defined as the distance between the points at which the conductance dropped below $1.2 G_{0}$ and $0.8 G_{0}$ for the $\mathrm{Au}$ junctions and $3.2 \times 10^{-3} G_{0}$ and 2.0 $\times 10^{-3} G_{0}$ for the molecular junctions, respectively. The average lengths for the Au junctions and the molecular junction were $0.078( \pm 0.008)$ and $0.073( \pm 0.007) \mathrm{nm}$, respectively. Two breakdown distances agreed well with each other.

The breakdown can take place at one of the three bonds, $\mathrm{Au}-\mathrm{CN}, \mathrm{Au}-\mathrm{Au}$, or an intramolecular bond in the $\mathrm{Au} / 1,4-$ diisocyanobenzene/Au molecular junction. The respective bonds could show different breakdown distances because of the difference in the bond strength. Comparable breakdown distances indicate that breakdown in the molecular junction took place at the $\mathrm{Au}-\mathrm{Au}$ bond rather than the $\mathrm{Au}-\mathrm{CN}$ bond, which is compelling proof that the $\mathrm{Au}-\mathrm{CN}$ bond was stronger than the $\mathrm{Au}-\mathrm{Au}$ metal bond in the molecular junction with the $\mathrm{Au}-\mathrm{CN}$ bond. In the case of the $\mathrm{Au} / 1,4-$ benzenedithiol/Au molecular junction, the breakdown distance $[0.10 \mathrm{~nm}$ in Fig. 2(c)] was also comparable to that of the $\mathrm{Au}$ junction, indicating that the $\mathrm{Au}-\mathrm{S}$ bond was also stronger than the $\mathrm{Au}-\mathrm{Au}$ bond. The relatively short length of the plateau $[0.02 \mathrm{~nm}$ see Fig. 2(e)] of the Au/1,4dicyanobenzene/Au junction indicates that the $\mathrm{Au}-\mathrm{NC}$ bond was weaker than the $\mathrm{Au}-\mathrm{Au}$ bond. Similar behavior was also observed in the system of 1,8-octanedithiol bonded to two $\mathrm{Au}$ electrodes by force measurement using atomic force microscopy. ${ }^{16}$ The force required to break the molecular junctions was close to that of the Au junctions, indicating that the strength of the $\mathrm{Au}-\mathrm{S}$ bond is higher than that of the $\mathrm{Au}-\mathrm{Au}$ metal bond.

A well-defined molecular junction with a strong $\mathrm{Au}-\mathrm{CN}$ bond was fabricated in the present study. As for the disubstituted benzene, the conductance of the molecular junction with the $\mathrm{Au}-\mathrm{CN}$ bond was comparable to that of the molecular junction with the Au-S bond. The energy of the molecular orbital varies with the linker group and the end group of the molecule. In the case of 1,4-diisocyanobenzene, the linker and end group are defined as benzene ring $\left(\mathrm{C}_{6} \mathrm{H}_{4}\right)$ and isocyanide $(\mathrm{CN})$, respectively. In some linker groups, the energy difference between the Fermi level of the metal electrodes and conduction orbital of the molecule could be smaller for isocyanide end groups than thiols. Since the bond strength of the metal-CN bond is comparable to that of the metal-S bond, the conductance of the molecular junction with the metal-CN bond could be higher than that of the molecular junction with the metal-S bond in some combinations of the metals and molecules. In developing an electronic device with a single molecule, it will be important to study electron transport through a single molecule with a high degree of functionality, such as in the photoswitching effect and single molecule magnetism. By using the best end group for the individual molecule, the performance of the device would be drastically improved. The isocyanide end group would be a suitable candidate.

In conclusion, we studied the conductance of a single 1,4-diisocyanobenzene, 1,4-dicyanobenzene, or 1,4benzenedithiol molecule bridging two $\mathrm{Au}$ electrodes. The conductance of the molecular junction with the $\mathrm{Au}-\mathrm{CN}$ bond was comparable to that of the molecular junction with the $\mathrm{Au}-\mathrm{S}$ bond. The plateau length analysis showed that the $\mathrm{Au}-\mathrm{CN}$ coordination bond was stronger than $\mathrm{Au}-\mathrm{Au}$ metal bond. The isocyanide end group is a promising alternative to the thiol.

This work was partially supported by a Grant-in-Aid for Scientific Research A (No. 16205026) and Grant-in-Aid for Scientific Research on Priority Areas (No. 17069001) from MEXT.

${ }^{1}$ M. A. Reed, C. Zhou, C. J. Muller, T. P. Burgin, and J. M. Tour, Science 278, 252 (1997).

${ }^{2}$ B. Xu and N. J. Tao, Science 301, 1221 (2003).

${ }^{3}$ J. Ulrich, D. Esrail, W. Pontius, L. Venkataraman, D. Millar, and L. H. Doerrer, J. Phys. Chem. B 110, 2462 (2006).

${ }^{4}$ X. Xiao, B. Xu, and N. J. Tao, Nano Lett. 4, 267 (2004).

${ }^{5}$ T. Tada and K. Yoshizawa, ChemPhysChem 12, 1035 (2002).

${ }^{6}$ D. L. DuBose, R. E. Robinson, T. C. Holovics, D. R. Moody, E. C. Weintrob, C. L. Berrie, and M. V. Barybin, Langmuir 22, 4599 (2006).

${ }^{7}$ K. Hara, K. Iwahashi, Y. Kanamori, S. Naito, S. Takakusagi, K. Uosaki, and M. Sawamura, Chem. Lett. 35, 870 (2006).

${ }^{8}$ J. M. Seminario, C. E. De La Cruz, and P. A. Derosa, J. Am. Chem. Soc. 123, 5616 (2001).

${ }^{9}$ J. Chen, L. C. Calvet, M. A. Reed, D. W. Carr, D. S. Grubisha, and D. W. Bennett, Chem. Phys. Lett. 313, 741 (1999).

${ }^{10}$ S. K. Chastain and W. R. Mason, Inorg. Chem. 21, 3717 (1982).

${ }^{11}$ M. Kiguchi, T. Konishi, and K. Murakoshi, Phys. Rev. B 73, 125406 (2006).

${ }^{12}$ I. D. Cunningham, G. J. Buist, and S. R. Arkle, J. Chem. Soc., Perkin Trans. 2, 589 (1999).

${ }^{13}$ J. He, O. Sankey, M. Lee, N. Tao, X. Lic, and S. Lindsay, Faraday Discuss. 131, 145 (2006)

${ }^{14}$ N. D. Lang and Ph. Avouris, Phys. Rev. B 64, 125323 (2001).

${ }^{15}$ Y. Xue and M. A. Ratner, Phys. Rev. B 69, 85403 (2004).

${ }^{16}$ B. Xu, X. Xiao, and N. J. Tao, J. Am. Chem. Soc. 125, 16164 (2003). 